\title{
openheart Bivalirudin versus heparin in primary PCI: clinical outcomes and cost analysis
}

\author{
Pierre Deharo, ${ }^{1,2}$ Thomas W Johnson, ${ }^{1}$ Hazim Rahbi, ${ }^{1}$ Raveen Kandan, ${ }^{1}$ \\ Ruth Bowles, ${ }^{1}$ Abdul Mozid, ${ }^{1}$ Stephen Dorman, ${ }^{1}$ Julian W Strange, ${ }^{1}$ \\ Andreas Baumbach ${ }^{1,3}$
}

\begin{abstract}
- Additional material is published online only. To view please visit the journal online (http://dx.doi.org/10.1136/ openhrt-2017-000767)
\end{abstract}

To cite: Deharo P, Johnson TW, Rahbi $\mathrm{H}$, et al. Bivalirudin versus heparin in primary PCl: clinical outcomes and cost analysis. Open Heart 2018;5:e000767. doi:10.1136/

openhrt-2017-000767

Received 19 December 2017 Revised 7 February 2018 Accepted 14 February 2018

Check for updates

${ }^{1}$ Cardiology, Bristol Heart Institute, Bristol, UK

${ }^{2}$ Hopital la Timone, Marseille, France

${ }^{3}$ Barts Health NHS Trust, London, UK

Correspondence to Professor Andreas Baumbach; baumbach.andreas@nhs.net

\section{ABSTRACT}

Background The evidence for benefits of bivalirudin over heparin has recently been challenged. We aimed to analyse the safety and cost-effectiveness following reintroduction of heparin instead of bivalirudin as the standard anticoagulation for primary percutaneous coronary intervention (PPCl) in a high-volume centre. Methods and results This analysis was an open-label, prospective registry including all patients admitted to our centre for PPCI from April 2014 to April 2016. Heparin was reintroduced as standard anticoagulant in April 2015. During the 2 years, 1291 patients underwent a PPCl, 662 in the Bivalirudin protocol period (Cohort B) and 629 in the Heparin protocol period (Cohort H). Baseline and procedural characteristics were not significantly different, except for a higher use of thromboaspiration and femoral access in the earlier Cohort B. Glycoprotein 2b3a (Gp2b3a) antagonists were used in $24 \%$ of the patients in Cohort $B$ versus $28 \%$ in Cohort $H(P<0.01)$. We did not observe any differences in death at 180 days $(11.03 \%$ in Cohort B vs $11.29 \%$ in Cohort $\mathrm{H})(\mathrm{HR} 95 \% \mathrm{Cl} 0.98$ (0.72 to 1.33 ), $\mathrm{P}=0.88)$. The incidence of any bleeding complications at 30 days did not differ between the two periods $(21.9 \%$ vs $21.9 \%, \mathrm{P}=0.99)$. The cost related to the anticoagulants amounted to $£ 246236$ in Cohort B versus $£ 4483$ in Cohort H ( $\_324406$ vs $£ 102347$ when adding Gp2b3a antagonists).

Conclusion We did not find clinically relevant changes in patient outcomes, including bleeding complications with reintroduction of heparin in our PPCI protocol. However, the use of heparin was associated with a major reduction in treatment costs.

\section{INTRODUCTION}

European and American guidelines recommend intravenous anticoagulation in all patients undergoing primary percutaneous coronary intervention (PPCI). ${ }^{12}$ Bivalirudin is a specific, reversible, direct thrombin inhibitor, characterised by a quick onset of action and short half-life, overcoming the limitations of heparin, with a more predictable antithrombotic response. Harmonizing Outcomes with Revascularization and Stents in Acute Myocardial Infarction (HORIZONS-AMI) and most recently the European Ambulance Acute Coronary Syndrome Angiography (EUROMAX) trial suggested the

\section{Key questions}

What is already known about this subject?

- Bivalirudin is associated with reduction in the risk of bleeding events during primary percutaneous coronary intervention (PPCl) for ST elevation myocardial infarction (STEMI) in comparison with heparin versus Gp2b3a inhibitors.

- Recently, similar outcomes between bivalirudin and heparin has been showed in randomized trials, with higher risk of stent thrombosis with bivalirudin.

What does this study add?

- The present analysis showed that the reintroduction of heparin instead of bivalirudin as standard anticoagulant for PPCI did not lead to significant differences in efficacy or safety outcomes, but was associated with a significant cost saving.

How might this impact on clinical practice?

- The use of heparin should be the first line anticoagulant during the management of STEMI with PPCl.

superiority of bivalirudin versus the combination of heparin plus glycoprotein 2b3a (Gp2b3a) antagonists in patients undergoing PPCI. The benefit was in net adverse clinical events, driven mainly by the reduction of bleeding complications, despite a higher rate of stent thrombosis (ST). ${ }^{34}$

Bivalirudin use in PPCI has recently been challenged by the results of the Unfractionated heparin versus bivalirudin in primary percutaneous coronary intervention (HEATPPCI) trial. This single-centre randomised trial compared bivalirudin and unfractionated heparin (UFH) with bailout Gp2b3a and favoured heparin with respect to ischaemic and bleeding outcomes. ${ }^{5}$ This trial used contemporary methods, including radial arterial access and more potent P2Y12 blockers (ie, prasugrel and ticagrelor). as the default strategy. As a result, the most recent guidelines of the European Society of Cardiology (ESC) downgraded the recommendation to 
use bivalirudin from IB to IIA. ${ }^{1}$ Following this, Bivalirudin or unfractionated heparin in patients with acute coronary syndromes managed invasively with and without ST elevation (MATRIX) trial showed in the largest and most contemporary cohort, similar outcomes between heparin and bivalirudin. ${ }^{6}$

Prior to publication of the HEAT-PPCI results, the standard of care at our institution was to use bivalirudin as the anticoagulant of choice for PPCI, unless contraindicated. Due to the changes in the ESC guidance plus the geographical and procedural similarities between our centre and the HEAT-PPCI study centre, we switched to heparin as our default antithrombotic agent. We prospectively assessed clinical outcomes, including bleeding complications and treatment costs.

The objective of the present study was to investigate the differences in clinical outcomes and financial costs following the reintroduction of heparin as the standard anticoagulant in patients treated for PPCI in our highvolume centre.

\section{MATERIALS AND METHODS \\ Study design and patients}

This analysis was an open-label, single-centre, prospective registry undertaken at the Bristol Heart Institute, Bristol, UK. All patients undergoing PPCI from April 2014 to April 2016 were prospectively enrolled.

Two periods were defined: Cohort B encompassed all PPCI patients admitted from 1 April 2014 to 30 March 2015. During this period, bivalirudin was used as the standard for anticoagulation in PPCI, unless contraindicated. Cohort $\mathrm{H}$ included patients treated by PPCI between 1 April 2015 and 1 April 2016 following an institutional protocol amendment resulting in the use of UFH as the standard periprocedural anticoagulant.

The indication for PPCI was based on the current guidance from the ESC. ${ }^{1}$ All patients, including out-of-hospital cardiac arrest, intubated patients and cardiogenic shock, were included in the main analysis. A prespecified analysis was planned in the cohort of non-ventilated patients.

\section{Treatment}

Bivalirudin was started as soon as possible during the PPCI and was given as a bolus of $0.75 \mathrm{mg}$ per $\mathrm{kg}$ of body weight, followed by an infusion of $1.75 \mathrm{mg}$ per $\mathrm{kg}$ per hour for 4 hours following the procedure. ${ }^{1}$ Heparin was given at a dosage of 70-100 IU per kg (operator preference) and then guided by activated clotting time (ACT) results, aiming to maintain an ACT of $>250 \mathrm{~s}$.

Bailout use of a Gp2b3a inhibitor was allowed in the event of high thrombus burden or microvascular obstruction (no reflow), as recommended in ESC guidelines. ${ }^{1}$ Any Gp2b3a inhibitor could be used at the approved doses and regimens.

During the index admission, a $300 \mathrm{mg}$ loading dose of aspirin was given to patients who were treatment naive before the study. All patients were pretreated with a loading dose of clopidogrel $600 \mathrm{mg}$, ticagrelor $180 \mathrm{mg}$ or prasugrel $60 \mathrm{mg}$ before or during the PPCI. The use of prasugrel and ticagrelor was recommended over clopidogrel unless contraindicated, but the choice was left to the treating physician. Other cardiac medications were given according to ESC guidelines. ${ }^{1}$

\section{Follow-up and endpoint assessments}

We aimed to assess the incidence of death, cerebrovascular accident (CVA), target lesion failure (TLF), definite ST and bleeding at 30 days. Additionally, the mortality rate was evaluated again at 6 months.

TLF was defined as any unexpected coronary revascularisation procedure (PCI or coronary artery bypass graft surgery) or reinfarction of the target lesion during the follow-up period. A diagnosis of CVA was made by a treating neurologist. CT or MRI was used to distinguish ischaemic from haemorrhagic strokes. Definite ST was classified according to the Academic Research Consortium criteria. ${ }^{7}$ Bleeding episodes were classified according to the Bleeding Academic Research Consortium (BARC) classification. ${ }^{8}$

All data were collected prospectively using standardised questionnaire and were entered into a central database. Follow-up was planned for 6 months after inclusion or until the time of death, whichever came first.

All events were adjudicated by a local research committee that was unaware of treatment allocations. All thrombotic and bleeding events requiring medical attention were verified by research nurses who were blinded to the patient's study group by use of medical records obtained from referring family doctors and hospitals.

\section{Cost analysis}

All costs were expressed in Great British Pound. The exact dosages of anticoagulants and Gp2b3a inhibitors administrated during the hospitalisation were reported, for each patient and procedure, by analysis of the medical and drug charts. We used the drug cost at our institution as primary base-case estimate: a $20 \mathrm{~mL}$ heparin phial costs $£ 4.20$, a bivalirudin $250 \mathrm{mg} / 5 \mathrm{~mL}$ phial costs $£ 372$, a $12.5 \mathrm{mg} / 50 \mathrm{~mL}$ tirofiban phial costs $£ 146.10$ and a $10 \mathrm{mg} / 5 \mathrm{~mL}$ abciximab phial costs $£ 265.60$. The other antithrombotic costs were assumed to be negligible and equal for all agents. We aimed to define the cost of antithrombotic agents associated with the PPCI index procedure.

\section{Statistical analysis}

All calculations were performed using SPSS software (version 20.00) and GraphPad prism (version 7.0). Continuous variables were reported as means and SD or as medians and range (according to their distribution), and categorical variables were reported as count and percentages. Standard two-sided tests were used to compare continuous characteristics (Student's t-tests or Mann-Whitney U-tests) or categorical characteristics $\left(\chi^{2}\right.$ or Fisher's exact tests) among patient groups. As a 
measure of strength of the treatment effect, we calculated HR and 95\% CI for 'Cohort B' versus 'Cohort H' by means of Cox's proportional hazards regression analysis.

\begin{tabular}{|c|c|c|c|}
\hline & $\begin{array}{l}\text { Cohort B } \\
(n=662)\end{array}$ & $\begin{array}{l}\text { Cohort H } \\
(n=629)\end{array}$ & $P$ value \\
\hline Age, mean (SD), years & $65.6 \pm 12.9$ & $64.6 \pm 13.3$ & 0.15 \\
\hline Body mass index, mean (SD) & $28.6 \pm 21.7$ & $28.5 \pm 17.3$ & 0.93 \\
\hline Male gender, $\mathrm{n}(\%)$ & $473(71.5 \%)$ & $483(76.8 \%)$ & 0.03 \\
\hline \multicolumn{4}{|l|}{ Medical history, n (\%) } \\
\hline Coronary artery disease & $152(23.0 \%)$ & $134(21.3 \%)$ & 0.47 \\
\hline Myocardial infarction & $113(17.1 \%)$ & $92(14.6 \%)$ & 0.23 \\
\hline CABG & $23(3.5 \%)$ & $20(3.2 \%)$ & 0.77 \\
\hline Coronary revascularisation & $88(13.3 \%)$ & $74(11.8 \%)$ & 0.41 \\
\hline Stroke/TIA > 1 year & $29(4.4 \%)$ & $16(2.5 \%)$ & 0.07 \\
\hline Peripheral arterial disease & $31(4.7 \%)$ & $25(4.0 \%)$ & 0.21 \\
\hline Congestive heart failure & $6(0.9 \%)$ & $7(1.1 \%)$ & 0.71 \\
\hline Hypertension & $354(53.5 \%)$ & $303(48.2 \%)$ & 0.06 \\
\hline Hypercholesterolaemia & $307(46.4 \%)$ & $294(46.7 \%)$ & 0.90 \\
\hline Diabetes & $101(15.3 \%)$ & $83(13.2 \%)$ & 0.29 \\
\hline Current smoker & $216(32.6 \%)$ & $248(39.4 \%)$ & 0.01 \\
\hline \multicolumn{4}{|l|}{ Long-term antiplatelet therapy, n (\%) } \\
\hline Aspirin & $256(38.7 \%)$ & $243(38.6 \%)$ & 0.99 \\
\hline \multicolumn{4}{|l|}{ EKG presentation, n (\%) } \\
\hline ST elevation & $640(96.7 \%)$ & $608(96.7 \%)$ & 0.99 \\
\hline LBBB & $10(1.5 \%)$ & $7(1.1 \%)$ & 0.53 \\
\hline \multicolumn{4}{|l|}{ EKG territory of infarction, n (\%) } \\
\hline Anterior & $223(33.7 \%)$ & $229(36.4 \%)$ & 0.31 \\
\hline Cardiogenic shock, n (\%) & $82(12.4 \%)$ & $77(12.2 \%)$ & 0.94 \\
\hline Ventilated patients, n (\%) & $59(8.9 \%)$ & $57(9.1 \%)$ & 0.93 \\
\hline Out-of-hospital cardiac arrest, n (\%) & $69(10.4 \%)$ & $66(10.5 \%)$ & 0.97 \\
\hline \multicolumn{4}{|l|}{ Anticoagulation, n (\%) } \\
\hline Bivalirudin & $498(75.2 \%)$ & $5(0.8 \%)$ & $<0.01$ \\
\hline Heparin & $164(24.8 \%)$ & $624(99.2 \%)$ & $<0.01$ \\
\hline Recent lysis, n (\%) & $1(0.2 \%)$ & $0(0 \%)$ & \\
\hline Gp2b3a agent, n (\%) & $154(22.3 \%)$ & $177(28.1 \%)$ & 0.05 \\
\hline Tirofiban & $38(5.7 \%)$ & $22(3.5 \%)$ & 0.06 \\
\hline Abciximab & $116(17.5 \%)$ & $155(24.6 \%)$ & $<0.01$ \\
\hline Loading dose of antiplatelet, $\mathrm{n}(\%)$ & $633(95.6 \%)$ & $611(97.1 \%)$ & 0.15 \\
\hline \multicolumn{4}{|c|}{ Antiplatelet therapy received between randomisation and discharge, $\mathrm{n}(\%)$} \\
\hline Aspirin & $652(98.5 \%)$ & $617(98.1 \%)$ & 0.58 \\
\hline \multicolumn{4}{|l|}{ Oral P2Y12 blocker, n (\%) } \\
\hline Clopidogrel & $134(20.2 \%)$ & $75(11.9 \%)$ & $<0.01$ \\
\hline Prasugrel & $411(62.1 \%)$ & $362(57.6 \%)$ & 0.10 \\
\hline Ticagrelor & $117(17.7 \%)$ & 192 (30.5\%) & $<0.01$ \\
\hline
\end{tabular}

CABG, coronary artery bypass graft; Gp2b3a, glycoprotein 2b3a; LBBB, left bundle branch block.; TIA, transient ischaemic attack. 
Table 2 Baseline procedural characteristics

\begin{tabular}{|c|c|c|c|}
\hline & $\begin{array}{l}\text { Cohort B } \\
(n=662)\end{array}$ & $\begin{array}{l}\text { Cohort H } \\
(n=629)\end{array}$ & $P$ value \\
\hline \multicolumn{4}{|l|}{ Access site, $\mathrm{n}(\%)$} \\
\hline Femoral & $131(19.8 \%)$ & $79(12.6 \%)$ & $<0.01$ \\
\hline Culprit lesion, $\mathrm{n}(\%)$ & & & 0.60 \\
\hline LMS & $16(2.2 \%)$ & $24(3.4 \%)$ & 0.18 \\
\hline LAD & $270(37.8 \%)$ & $272(38.9 \%)$ & 0.91 \\
\hline $\mathrm{LCx}$ & $127(17.8 \%)$ & $126(18.0 \%)$ & 0.92 \\
\hline RCA & $289(40.5 \%)$ & $264(37.7 \%)$ & 0.29 \\
\hline Graft & $12(1.7 \%)$ & $14(2.0 \%)$ & 0.66 \\
\hline Number of bystander lesions $(\mathrm{n} \%)^{*}$ & $390(35.3 \%)$ & $308(30.6 \%)$ & 0.02 \\
\hline Number of lesions attempted $(\mathrm{n} \%)^{*}$ & $822(74.5 \%)$ & $834(82.7 \%)$ & $<0.01$ \\
\hline Number of stents per patient, mean (SD) & $1.3 \pm 0.8$ & $1.4 \pm 0.9$ & 0.01 \\
\hline Drug eluting stent, $(\mathrm{n} \%) \dagger$ & $815(95.5 \%)$ & $866(97.6 \%)$ & 0.02 \\
\hline Stent diameter, mean (SD) & $3.3 \pm 0.7$ & $3.4 \pm 1.5$ & 0.11 \\
\hline Stent length, mean (SD) & $27.1 \pm 10.2$ & $27.8 \pm 11.2$ & 0.09 \\
\hline TIMI flow in the culprit artery at the end of the procedure, $n(\%) \neq$ : & & & 0.17 \\
\hline $0-1$ & $20(2.8 \%)$ & $12(1.7 \%)$ & \\
\hline $2-3$ & $694(97.2 \%)$ & $688(98.3 \%)$ & \\
\hline Number of lesions successful, $n(\%) \S$ : & $793(96.5 \%)$ & $799(95.8 \%)$ & 0.48 \\
\hline Use of a thrombus extraction catheter, $n(\%)$ : & $297(44.9 \%)$ & $78(12.4 \%)$ & $<0.01$ \\
\hline Use of a vascular closure device system, $n(\%) \eta$ : & $107(81.7 \%)$ & $76(96.2 \%)$ & $<0.01$ \\
\hline Thrombotic procedural complications, $n(\%)$ : & $21(3.17 \%)$ & $13(2.07 \%)$ & 0.22 \\
\hline
\end{tabular}

If more than one stent is used, the stent length denotes the sum of all stent lengths.

LAD, left anterior descending coronary artery; LCx, left circumflex coronary artery; LMS, left main stem; RCA,right coronary artery.

\section{RESULTS}

\section{Baseline}

Between April 2014 and April 2016, we enrolled 1291 patients, of whom 662 were included in Cohort B and 629 in Cohort H. Baseline and procedural characteristics are presented in tables 1 and 2. In Cohort B, $75.2 \%$ of the patients were treated with bivalirudin while $24.8 \%$ received heparin. In Cohort H, 99.2\% of patients had heparin and $0.8 \%$ received bivalirudin. One thousand one hundred and seventy-five $(91.0 \%)$ patients were enrolled in the non-ventilated cohort, corresponding to 603 in Cohort B and 572 in Cohort H.

Regarding the antiplatelet strategy, we observed that clopidogrel was less frequently used in Cohort $\mathrm{H}(11.9 \%$ vs $20.2 \%, \mathrm{P}<0.01)$. The use of Gp2b3a inhibitors was higher in Cohort H (28.1\% vs $22.3 \%, \mathrm{P}=0.05)$, mostly driven by more frequent use of abciximab $(24.6 \%$ vs $17.5 \%, \mathrm{P}<0.01)$.

Regarding the procedural characteristics, the femoral access was less frequently used in Cohort $\mathrm{H}(12.6 \%$ vs $19.8 \%, \mathrm{P}<0.01)$. We observed significant difference in the use of mechanical thrombus aspiration with a significant drop in the later Cohort $\mathrm{H}(44.9 \%$ vs $12.4 \%, \mathrm{P}<0.01)$.

\section{Endpoints}

At 30 days, any ischaemic endpoint occurred in 72 $(10.9 \%)$ patients in Cohort B and in $67(10.7 \%)$ in Cohort H (HR 95\% CI 1.02 (0.72 to 1.46), $\mathrm{P}=0.90$ ).

At 30 days, $62(9.4 \%)$ patients died in Cohort B versus $56(8.9 \%)$ in Cohort H (HR 95\% CI 1.05 (0.75 to 1.49), $\mathrm{P}=0.77$ ) (table 3 , figure 1). At 180 days, 73 (11.03\%) patients died in Cohort B versus $71(11.3 \%)$ in Cohort H (HR 95\% CI 0.98 (0.72 to 1.33), $\mathrm{P}=0.88$ ) (table 3, figure 1).

TLF was observed at 30 days in 12 (1.8\%) patients in Cohort B and $11(1.8 \%)$ in Cohort H (HR 95\% CI 1.03. (0.46 to 2.33), $\mathrm{P}=0.93$ ); a CVA event at 30 days was observed in five $(0.8 \%)$ patients in Cohort $\mathrm{B}$ and three (0.5\%) patients in Cohort H (HR 95\% CI 1.58 (0.38 to 6.60 ), $\mathrm{P}=0.52$ ) (table 3 , figure 1 ). The rate of definite $\mathrm{ST}$ at 30 days was low and not different between the two groups (three $(0.5 \%)$ patients in Cohort B vs two $(0.3 \%)$ in Cohort H, $\mathrm{P}=0.70)$.

Bleeding events defined as all BARC occurred in 145 (21.9\%) patients in Cohort B and in 138 (21.9\%) in Cohort H (HR 95\% CI 0.99 (0.91 to 1.23), $\mathrm{P}=0.99$ ) (table 3, figure 2). Bleeding events defined as BARC $\geq 2$ occurred in 102 (15.4\%) patients in Cohort B and in 115 
Table 3 Endpoints at 30 and 180 days in the whole cohort

\begin{tabular}{llll}
\hline & Cohort B & Cohort H & P value \\
\hline Death 30 days & $62(9.4 \%)$ & $56(8.9 \%)$ & 0.77 \\
\hline Death 180 days & $73(11.0 \%)$ & $71(11.3 \%)$ & 0.88 \\
CVA 30 days & $5(0.8 \%)$ & $3(0.5 \%)$ & 0.52 \\
\hline TLF 30 days & $12(1.8 \%)$ & $11(1.8 \%)$ & 0.93 \\
\hline Definite ST 30 days & $3(0.5 \%)$ & $2(0.3 \%)$ & 0.70 \\
Bleeding all 30 days & $145(21.9 \%)$ & $138(21.9 \%)$ & 0.99 \\
Bleeding BARC $\geq 230$ days & $102(15.4 \%)$ & $115(18.3 \%)$ & 0.17 \\
Bleeding BARC 2 30 days & $82(12.4 \%)$ & $99(15.7 \%)$ & 0.09 \\
Bleeding BARC 3 30 days & $17(2.6 \%)$ & $15(2.4 \%)$ & 0.83 \\
Bleeding BARC 4 30 days & 1 & 0 & - \\
Bleeding BARC 5 30 days & 2 & 1 & - \\
\hline
\end{tabular}

CVA, cerebrovascular accident; ST, stent thrombosis; TLF, target lesion failure.

patients $(18.3 \%)$ in Cohort H (HR 95\% CI 0.84 (0.66 to 1.08), $\mathrm{P}=0.17)$.

When we directly compare heparin versus bivalirudin during the whole period, any ischaemic endpoint occurred in $89(11.2 \%)$ patients on heparin and in 50 (9.9\%) on bivalirudin (HR 95\% CI 1.14 (0.82 to 1.56), $\mathrm{P}=0.44)$ at 30 days. Bleeding all BARC was observed in 180 patients $(22.8 \%)$ on heparin versus $103(20.5 \%)$ on bivalirudin (HR 95\% CI 1.12 (0.90 to 1.38), $\mathrm{P}=0.32$ ), while bleeding BARC 2-5 was observed in $150(19.0 \%)$ versus $67(13.3 \%)$ patients (HR 95\% CI 1.43 (1.10 to 1.86), $\mathrm{P}=0.001)$.

When the analysis was restricted to non-ventilated patients, we observed that at 30 days $36(6.0 \%)$ patients died in Cohort B versus $30(5.2 \%)$ in Cohort H (HR $95 \%$ CI 1.14 (0.71 to 1.82$), \mathrm{P}=0.59$ ). At 180 days, 44 (7.3\%) patients died in Cohort B versus $43(7.5 \%)$ in Cohort $\mathrm{H}$ (HR 95\% CI 0.97 (0.65 to 1.46), $\mathrm{P}=0.89$ ) (table 4).

TLF at 30 days was observed in $12(2.0 \%)$ patients in Cohort B and $9(1.6 \%)$ in Cohort H (HR 95\% CI 1.27 (0.53 to 2.98), $\mathrm{P}=0.59)$; a CVA event at 30 days was observed in one $(0.2 \%)$ patient in Cohort $\mathrm{B}$ and two (0.4\%) patients in Cohort H (HR 95\% CI 0.47 (0.04 to 5.22), $\mathrm{P}=0.53$.). Definite $\mathrm{ST}$ at 30 days was reported in three patients in Cohort B versus one in Cohort $\mathrm{H}$ $(\mathrm{P}=0.34)$. Bleeding events defined as all BARC occurred in $132(21.9 \%)$ patients in Cohort B and in $114(19.9 \%)$ in Cohort H (HR 95\% CI 1.21 (0.97 to 1.69), $\mathrm{P}=0.10$ ) (table 4). Bleeding events defined as BARC $\geq 2$ occurred in $91(15.1 \%)$ patients in Cohort B and in 92 patients (16.1\%) in Cohort H (HR 95\% CI 0.94 (0.72 to 1.23), $\mathrm{P}=0.64)$.

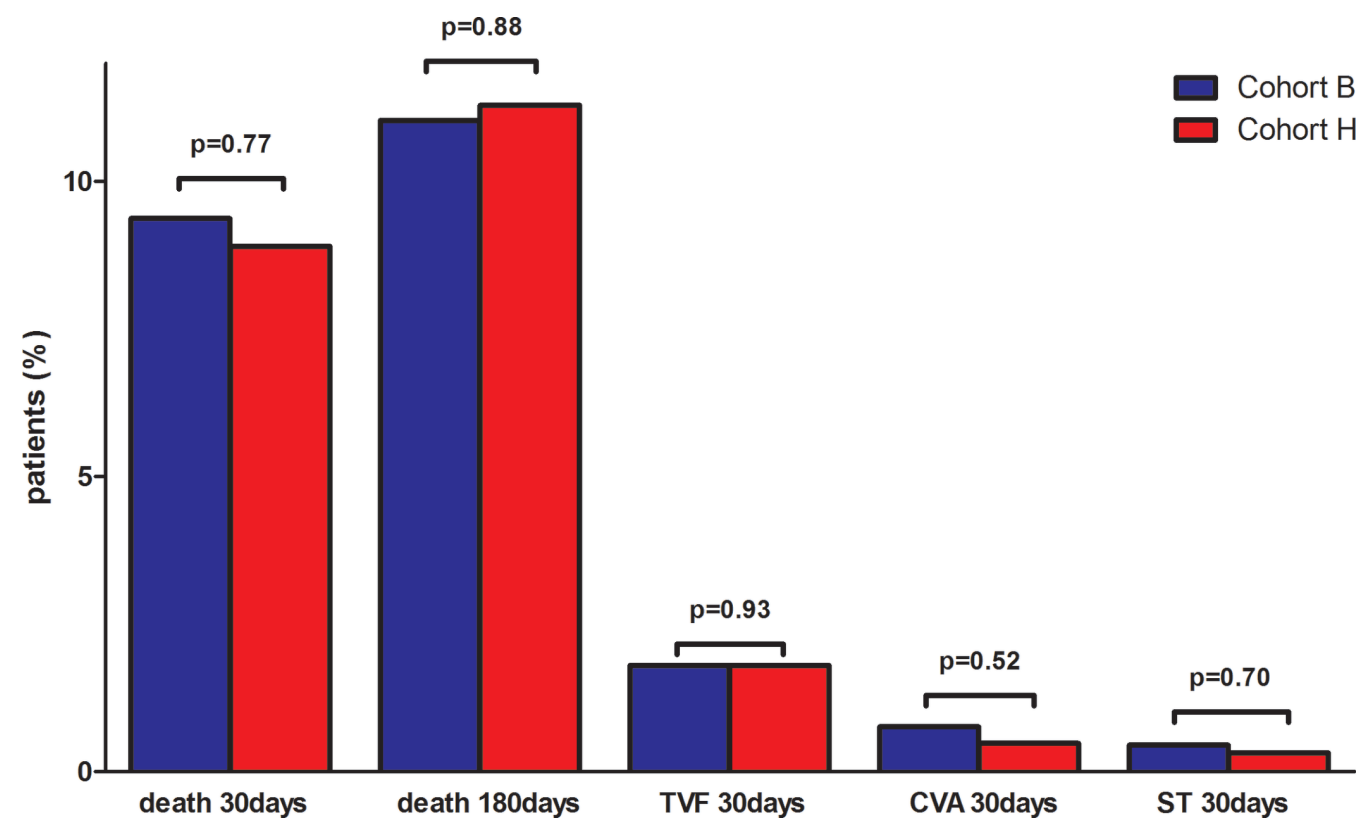

Figure 1 Incidence of ischaemic endpoints at 30 and 180 days. CVA, cerebrovascular accident; ST, stent thrombosis; TVF, target vessel failure. 


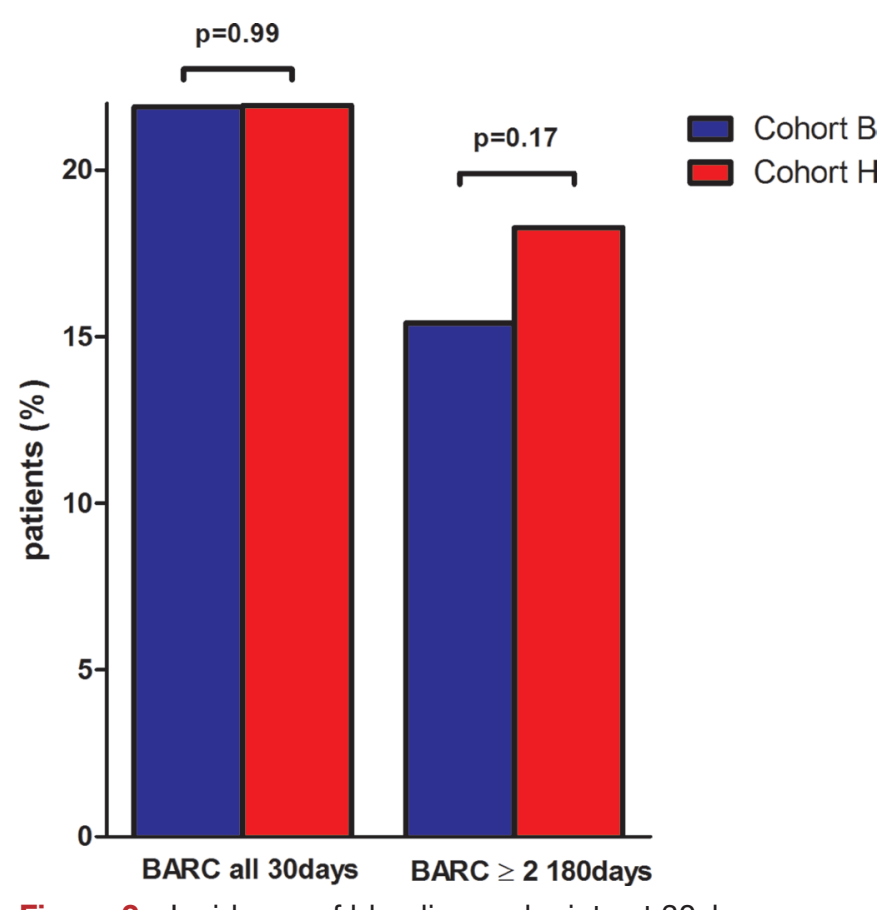

Figure 2 Incidence of bleeding endpoints at 30 days.

\section{Cost analysis}

The total costs related to the anticoagulants used for the index procedure in Cohort B were $£ 246236.0$ versus $£ 4483.0$ in Cohort $\mathrm{H}(\mathrm{P}<0.01)$, corresponding to $£ 372.0$ per patient versus $£ 7.1$ per patient (figure $3 \mathrm{~A}$ ). When we added the cost of Gp2b3a inhibitors, the difference remained significant with $£ 324406.0$ in Cohort B versus $£ 102347.0$ in Cohort $\mathrm{H} \quad(\mathrm{P}<0.01)$, corresponding to $£ 490.0$ per patient versus $£ 162.7$ per patient (figure $3 \mathrm{~B}$ ).

\section{DISCUSSION}

We report 'real-world' adoption of the latest evidence in acute antithrombotic therapy in PPCI and have demonstrated significant cost savings through the reintroduction of UFH use with comparable ischaemic and bleeding outcomes.

HORIZONS-AMI was the first randomised trial to show the superiority of bivalirudin (administered without a post-PPCI infusion) versus heparin plus Gp2b3a, in patients undergoing PPCI, on net adverse clinical events, driven mostly by the reduction of bleeding complications. ${ }^{3}$ More recently, EUROMAX confirmed the reduction in the composite endpoint of bleeding plus death with bivalirudin (administered with a post-PPCI infusion) versus heparin plus optional Gp2b3a antagonists $(11.5 \%$ vs $69 \%$ of patients had Gp2b3a inhibitors, respectively). ${ }^{4}$ A higher rate of ST was observed with use of bivalirudin in both trials. ${ }^{34}$ HORIZONS-AMI demonstrated a mortality benefit with bivalirudin use, which was not confirmed in EUROMAX. In contrast, HEAT-PPCI, a single- centre randomised trial, investigated bivalirudin (without post-procedure infusion) versus low-dose heparin (70 IU per $\mathrm{kg}$ ), limiting Gp2b3a use to bailout in both groups (13\% in each group). ${ }^{5}$ The incidence of the primary ischaemic endpoint (all-cause mortality, stroke, recurrent infarction and unplanned target lesion revascularisation) was higher in the bivalirudin group. Interestingly, bivalirudin failed to prove a significant reduction in bleeding complications $(12.5 \%$ vs $13.5 \%, \mathrm{P}=0.54)$.

Since then, Bavarian Reperfusion Alternatives Evaluation 4 trial compared prasugrel plus bivalirudin versus clopidogrel plus heparin in PPCI and was stopped prematurely due to slow recruitment and failure to demonstrate differences in outcome. ${ }^{9}$ The BivaliRudin in Acute Myocardial Infarction vs Glycoprotein IIb/IIIa and Heparin: a Randomised Controlled Trial, a Chinese randomised trial, compared bivalirudin versus heparin plus Gp2b3a in PPCI, with a reduction of net adverse clinical events with bivalirudin. ${ }^{10}$ Most recently, the MATRIX randomised trial showed in 7213 patients with acute coronary syndrome (ACS) (of whom 55.6\% underwent PPCI) no differences between bivalirudin and heparin

\begin{tabular}{|c|c|c|c|}
\hline & Cohort B & Cohort H & $P$ value \\
\hline Death 30 days & $36(6.0 \%)$ & $30(5.2 \%)$ & 0.59 \\
\hline Death 180 days & $44(7.3 \%)$ & $43(7.5 \%)$ & 0.89 \\
\hline CVA 30 days & $1(0.2 \%)$ & $2(0.4 \%)$ & 0.53 \\
\hline TLF 30 days & $12(2.0 \%)$ & $9(1.6 \%)$ & 0.59 \\
\hline Definite ST 30 days & $3(0.5 \%)$ & $1(0.2 \%)$ & 0.34 \\
\hline Bleeding all 30 days & $132(21.9 \%)$ & $114(17.9 \%)$ & 0.10 \\
\hline Bleeding $B A R C \geq 230$ days & $91(15.1 \%)$ & $92(16.1 \%)$ & 0.64 \\
\hline Bleeding BARC 230 days & $78(12.9 \%)$ & $83(14.5 \%)$ & 0.09 \\
\hline Bleeding BARC 330 days & $10(1.7 \%)$ & $9(1.6 \%)$ & 0.83 \\
\hline Bleeding BARC 430 days & 1 & 0 & - \\
\hline Bleeding BARC 530 days & 2 & 0 & - \\
\hline
\end{tabular}

CVA, cerebrovascular accident; ST, stent thrombosis; TLF, target lesion failure. 
A

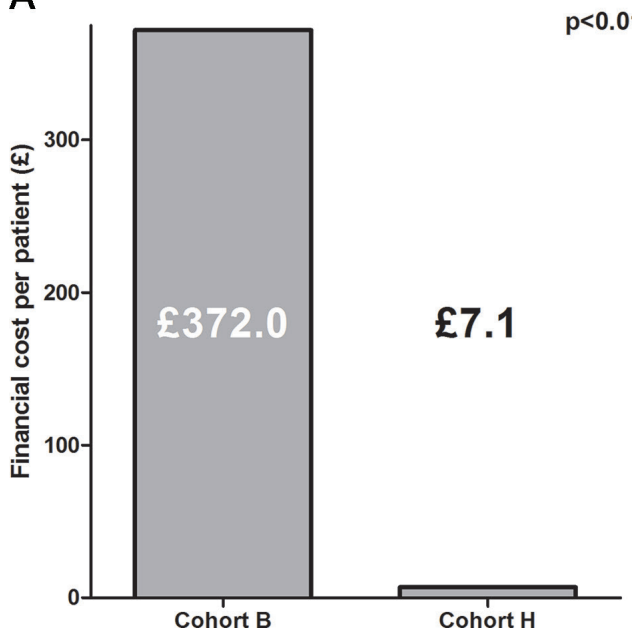

B

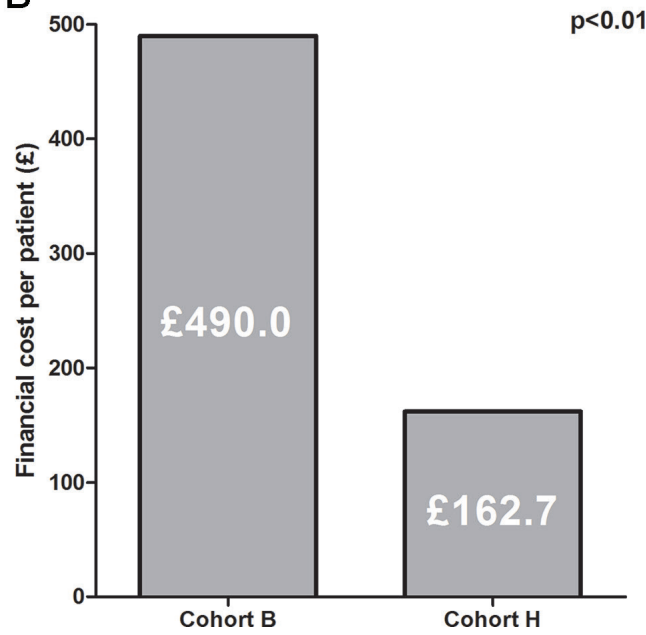

Figure 3 (A) Procedural anticoagulant cost analysis. (B) Procedural anticoagulants and glycoprotein 2b3a (Gp2b3a) inhibitors cost analysis.

regarding the ischaemic outcomes, while bivalirudin significantly reduced the bleeding complication rates. ${ }^{6}$

The conflicting results of these studies, complexity of study designs, with mandatory/discretionary use of Gp2b3a inhibitors, variations in bivalirudin protocols and geographical differences in clinical practice (particularly radial access use) have led to confusion surrounding the optimal antithrombotic regime for PPCI. However, the results of HEAT-PPCI were embraced by UK interventional cardiologists and have resulted in significant reductions in the use of bivalirudin for PPCI. Our results support the results observed by the HEATPPCI investigators with no observed differences in ischaemic or bleeding endpoints with use of heparin versus bivalirudin. However, we noticed that the reintroduction of UFH was associated with an increased use of bailout Gp2b3a inhibitors compared with routine use of bivalirudin with a post-PCI infusion. This finding may have been accentuated by the significant reduction in the use of thromboaspiration catheters and potential increased presence of thrombus between cohorts, precipitated by the reporting of the findings of the The Trial of Routine Aspiration Thrombectomy with PCI versus PCI Alone in Patients with STEMI (TOTAL) study ${ }^{11}$ contributing to the increase of bailout Gp2b3a antagonists use.

The increase in ST incidence observed in HORIZONS-AMI and EUROMAX was confirmed in HEATPPCI (3, 4 and 5). Our local approach of administering bivalirudin as a bolus followed by a prolonged post-PPCI infusion was associated with very low levels of definite ST, with no significant difference observed in comparison with UFH. Interestingly, in the prespecified analysis of the MATRIX cohort including $55.6 \%$ of PPCI, the prolonged infusion did not impact the ST risk in comparison with no post-PPCI infusion. ${ }^{6}$

Nowadays, prasugrel and ticagrelor are the recommended first-line antiplatelet agents in PPCI, in association with aspirin and intravenous anticoagulants. ${ }^{1}$ Trial to Assess Improvement in Therapeutic Outcomes by Optimizing Platelet Inhibition with Prasugrel Thrombolysis in Myocardial Infarction and PLATelet inhibition and patient Outcomes trials proved their superiority over clopidogrel in reducing ischaemic complications. ${ }^{12}{ }^{13}$ However, enhanced potency of antiplatelet effect is associated with an increase in bleeding risk. ${ }^{12} 13$ The initial benefit of bivalirudin was proved mostly in clopidogrel-treated patients. ${ }^{34}$ Our modern cohort used preferentially the newer P2Y12 inhibitors in PPCI and confirmed that even if numerically lower rate of bleeding complications was observed in the bivalirudin period, no significant difference was shown between both protocols despite a very high use of prasugrel and ticagrelor.

Nowadays, cost-effectivenes of the procedures has to be considered. The incidence of STEMI requiring PPCI remains stable and remains a significant challenge in health systems. ${ }^{12}$ Bivalirudin has been proved to be cost-effective in comparison with heparin plus systematic Gp2b3a antagonist in PPCI setting. ${ }^{14}$ Recent changes in PPCI treatment, including high rate of radial access and bailout Gp2b3a use, have highlighted the need to re-evaluate the cost-effectiveness of these strategies. We confirmed that the reintroduction of heparin as standard anticoagulant for PPCI was associated with significant cost saving that was maintained despite a slightly higher rate of Gp2b3a antagonist use. Additionally, clinical outcomes were not influenced by the anticoagulant strategy.

\section{Limitations}

The present study has some limitations. First, the intrinsic limitations of this study result from the observational character of a prospective registry, with unadjusted baseline differences existing between the two cohorts. The two groups compared were treated at different time points, with the Heparin group $\mathrm{H}$ the year after the Bivalirudin group B. In the group B, a significant proportion of patients were treated with heparin as per individual treatment choice in higher risk patients. Secondarily, we observed higher rates of radial access and newer P2Y12 
blockers use in the later cohort, likely related to progressive adoption of PPCI recommendations. ${ }^{1}{ }^{2}$ Vascular closure systems being largely used nowadays when femoral access is needed and the decrease in terms of use of femoral route may have impacted the cost of the procedure. However, this cost is not dependent on the subject of interest for this analysis (ie, periprocedural antithrombotic strategy). Similarly, the decrease in the use of clopidogrel and parallel increase in the use of ticagrelor have an obvious impact in terms of cost but only when extrapolated to 1-year treatment cost. When focusing on the procedure, it does not appear to be relevant. Therefore, we decided not to include those prices in the analysis. Third, we included all PPCI patients referred to our centre, which include a substantial number of patients with cardiogenic shock and comatose patients. This explains the high rates of death at 6 months, compared with clinical trials. Fourth, major changes in PPCI techniques occurred during the planned follow-up period. TOTAL results confirmed the lack of benefit associated with manual thromboaspiration devices in PPCI, explaining the significant differences in use of this technique between the two cohorts. Finally, we were not able to provide exact duration of bivalirudin infusion for each patient. The recommended protocol was an infusion of 3 -4 hours post PPCI.

\section{CONCLUSION}

In conclusion, in the present analysis, we observed that the reintroduction of heparin instead of bivalirudin as standard anticoagulant for PPCI did not lead to significant differences in efficacy or safety outcomes, but was associated with a significant cost saving.

Acknowledgements To our nurses' team and technicians in executing this study.

Contributors PD, HR, RK, RB, AM and JWS report no conflict of interest. TWJ reports lecture and advisory fees from AstraZeneca, Daiichi Sankyo, advisory fees from Correvio and research and lecture fees from The Medicines Co. AB reports speaker fees from the Medicines Company and Abbott Vascular.

Funding This work was supported by Bristol Heart Institute.

Competing interests TWJ reports Lecture and advisory fees from AstraZeneca, Daiichi Sankyo, advisory fees from Correvio, and research and lecture fees from The Medicines $\mathrm{Co}$. AB reports speaker fees from the Medicines Company and Abbott Vascular.

Patient consent Not required.

Ethics approval The authors honoured the ethical principles for medical research involving human subjects as set out in the Declaration of Helsinki. They obtained clearance and approval from their institution's audit committee to use the data for this purpose. The data management and statistical analysis were performed by the research and development section, Bristol Heart Institute, Bristol, UK.

Provenance and peer review Not commissioned; externally peer reviewed.
Data sharing statement No additional data are available.

Open Access This is an Open Access article distributed in accordance with the Creative Commons Attribution Non Commercial (CC BY-NC 4.0) license, which permits others to distribute, remix, adapt, build upon this work non-commercially, and license their derivative works on different terms, provided the original work is properly cited and the use is non-commercial. See: http://creativecommons.org/ licenses/by-nc/4.0/

(c) Article author(s) (or their employer(s) unless otherwise stated in the text of the article) 2018. All rights reserved. No commercial use is permitted unless otherwise expressly granted.

\section{REFERENCES}

1. Windecker S, Kolh P, Alfonso F, et al. 2014 ESC/EACTS Guidelines on myocardial revascularization: The Task Force on Myocardial Revascularization of the European Society of Cardiology (ESC) and the European Association for Cardio-Thoracic Surgery (EACTS) Developed with the special contribution of the European Association of Percutaneous Cardiovascular Interventions (EAPCI). Eur Heart $J$ 2014;35:2541-619.

2. ACC/AHA/SCAI Focused Update on Primary Percutaneous Coronary Intervention for Patients With ST-Elevation Myocardial Infarction: An Update of the 2011 ACCF/AHA/SCAI Guideline for Percutaneous Coronary Intervention and the 2013 ACCF/AHA Guideline for the Management of ST-Elevation Myocardial Infarction: A Report of the American College of Cardiology/American Heart Association Task Force on Clinical Practice Guidelines and the Society for Cardiovascular Angiography and Interventions. Circulation 2015;2016:1135-47.

3. Stone GW, Witzenbichler B, Guagliumi G, et al. Bivalirudin during primary $\mathrm{PCl}$ in acute myocardial infarction. $N$ Engl J Med 2008;358:2218-30.

4. Steg PG, van 't Hof A, Hamm CW, et al. Bivalirudin started during emergency transport for primary PCI. N Engl J Med 2013;369:2207-17.

5. Shahzad A, Kemp I, Mars C, et al. Unfractionated heparin versus bivalirudin in primary percutaneous coronary intervention (HEATPPCI): an open-label, single centre, randomised controlled trial. Lancet 2014;384:1849-58.

6. Valgimigli M, Frigoli E, Leonardi S, et al. Bivalirudin or unfractionated heparin in acute coronary syndromes. N Engl J Med 2015;373:997-1009.

7. Cutlip DE, Windecker S, Mehran R, et al. Clinical end points in coronary stent trials: a case for standardized definitions. Circulation 2007; 115:2344-51.

8. Mehran R, Rao SV, Bhatt DL, et al. Standardized bleeding definitions for cardiovascular clinical trials: a consensus report from the Bleeding Academic Research Consortium. Circulation 2011;123:2736-47.

9. Schulz S, Richardt G, Laugwitz KL, et al. Prasugrel plus bivalirudin vs. clopidogrel plus heparin in patients with ST-segment elevation myocardial infarction. Eur Heart J 2014;35:2285-94.

10. Han Y, Guo J, Zheng Y, et al. Bivalirudin vs heparin with or without tirofiban during primary percutaneous coronary intervention in acute myocardial infarction: the BRIGHT randomized clinical trial. JAMA 2015;313:1336-46.

11. Jolly SS, Cairns JA, Yusuf S, et al. Randomized trial of primary $\mathrm{PCl}$ with or without routine manual thrombectomy. $\mathrm{N}$ Engl J Med 2015;372:1389-98.

12. Wiviott SD, Braunwald E, McCabe $\mathrm{CH}$, et al. Prasugrel versus clopidogrel in patients with acute coronary syndromes. N Engl J Med 2007;357:2001-15

13. Wallentin L, Becker RC, Budaj A, et al. Ticagrelor versus clopidogrel in patients with acute coronary syndromes. $N$ Engl $\mathrm{J}$ Med 2009;361:1045-57.

14. Schwenkglenks M, Toward TJ, Plent S, et al. Cost-effectiveness of bivalirudin versus heparin plus glycoprotein Ilb/IIla inhibitor in the treatment of acute ST-segment elevation myocardial infarction. Heart 2012;98:544-51. 